\title{
EXTRA-ABDOMINAL MUSCULO-APONEUROTIC FIBROMATOSIS: A CASE WITH METACHRONOUS MULTICENTRIC LESIONS OF THE RIGHT THIGH
}

\author{
Savelina L. Popovska, \\ Tatyana M. Betova, \\ Krasimir T. Petrov \\ Department of Pathology, \\ University Hospital - Pleven
}

Corresponding Author:

Savelina L. Popovska

e-mail: spopovska@yahoo.com

Received: September 28, 2014

Revision received: October 28, 2014

Accepted: November 24, 2014

\begin{abstract}
Summary
Fibromatoses are a group of benign proliferations of fibrous tissue with clinical behaviour ranging from that of truly malignant tumours to that of benign reactive fibrous proliferations. Some of the superficial fibromatoses are fairly common but deep ones, also known as desmoid tumours or musculoaponeurotic fibromatoses, are rare. We describe a case of extra-abdominal musculoaponeurotic fibromatosis in a 22 -years old male. He underwent 14 operations for a period of 9 years for fibromatosis of the posterior surface of the right lower limb. The histological findings of the lesions were similar consistent across surgeries and consistent with a diagnosis of aggressive extra-abdominal fibromatosis.
\end{abstract}

Key words: musculoaponeurotic fibromatosis, aggressive fibromatosis, deep extra-abdominal fibromatosis

\section{Introduction}

The term aggressive fibromatosis (AF) refers to a large group of benign fibrous tissue proliferations of similar microscopic appearance that in their biological behaviour are intermediate between benign fibrous tissues and fibrosarcomas [1].

The term "desmoid" derives from the Greek word "desmos" meaning band or tendon and was coined by Mueller in 1836 [2].

The first description of a desmoid tumour is, however, credited to Mac Farlane in 1832 [3]. AF is a relatively rare lesion representing less than $3 \%$ of all soft tissue tumours with a reported annual incidence of $0.2-0.5$ per 100,000 population $[4,5]$.

Young adults are most commonly affected, with peak prevalence between the ages of 25 and 35 years. Lesions in patients younger than 10 years are uncommon[5].

These types of fibromatoses most frequently occur at the torso and extremities. The latter localization accounts for approximately $70 \%$ of all 
cases. Frequently affected sites include the shoulder $(20 \%)$, the chest wall and the back $(15 \%)$, the thigh $(12 \%)$, the mesentery $(10 \%)$, the neck $(10 \%)$, and the knee $(7 \%)[6,7]$.

Although desmoid tumors most commonly arise from the rectus abdominis muscle in postpartum women and in scars due to abdominal surgery, they may arise in any skeletal muscle.

\section{Case description}

A twenty-two-year-old patient presented with a history of recurrent multiple swellings of the right thigh. He underwent 14 operations for a period of 9 years for fibromatosis of posterior surface of the right lower limb.

At the time of the first surgery, the tumor was sized $20 \times 15 \times 15 \mathrm{~cm}$ and was found mostly in the gluteal region, enveloping the ischiadic nerve without infiltration of its nerve sheath.

Out of the total amount of 34 nodules removed, 21 were removed from the thigh and 13 -from the calf region. The myotendinous junctions were exclusively involved.

Each recurrence involved new areas, not affected on previous occasions.

On examination, there was a localized or more diffuse enlargement of the thigh muscles. The swellings were firm to bony hard in consistency but usually painless.

Soft plain films revealed a slight distortion of the outlines and fat planes of the affected muscle group. The underlying bones were normal.

On MRI the affected limb presented with multicentre superficial or deep-seated lesions, some of them well-defined, others-ill-defined, with a slight distortion of the anatomy of the postero-lateral muscle groups, due to multiple previous surgeries. The lesions (involving mostly the myotendinous junctions) were hypointense on T1WI to the affected muscles above and below the knee. On T2WI and IR (with fat suppression) the nodules were hyperintense.

Conclusion: A case of multicentre extraabdominal desmoids recurrence.

\section{Grossly}

The formation was found very firm and white with whorled appearance. The tumour measured $15 \times 7 \times 5.5 \mathrm{~cm}$ (Fig. 1).

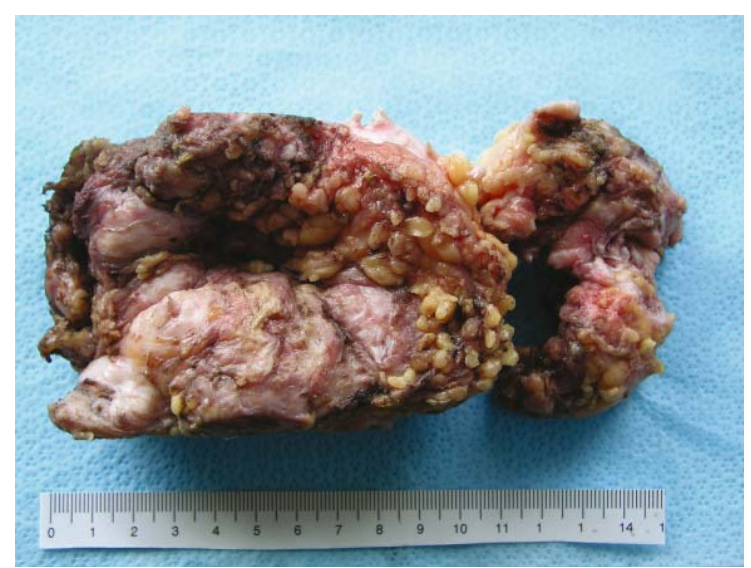

Figure 1. Macroscopic appearance of the lesion

\section{Microscopic examination}

Under the microscope the lesion was welldifferentiated and infiltrated the adjacent tissue. Microscopic examination showed interlacing bundles of uniform spindle-shaped cells with pale oval nuclei and tails of eosinophilic cytoplasm arranged in streaming fascicles. Some cells had inconspicuous nucleoli but there was no hyperchromasia or mitotic figures. There was a prominent collagen component seen as broad collagen bands.

The degree of cellularity was variable - some areas demonstrating a moderate number of lesional cells in the background stroma of abundant mature collagen, while others showed minimal stroma with a large number of active mesenchymal cells (Figures 2 and 3). The cellularity was most evident at the periphery of the tumour (Fig. 4).

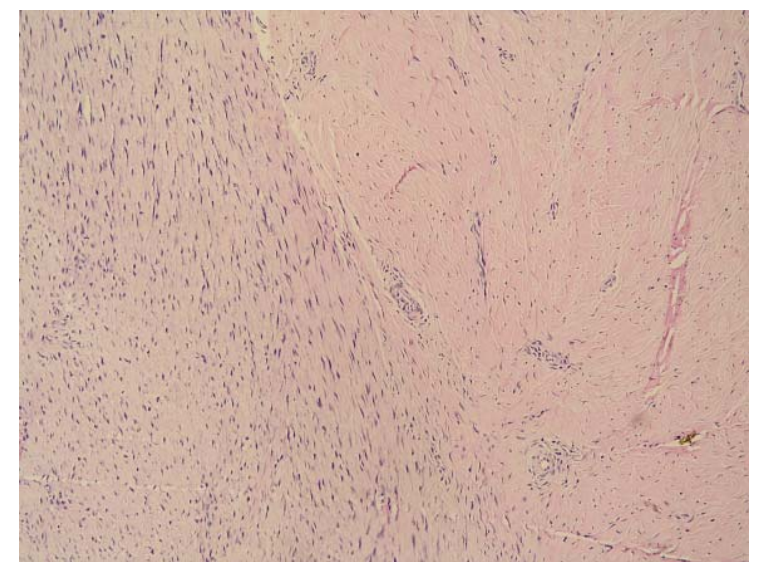

Figure 2. Microscopic appearance of the lesionH\&E staining, orig. magn. 10x20 


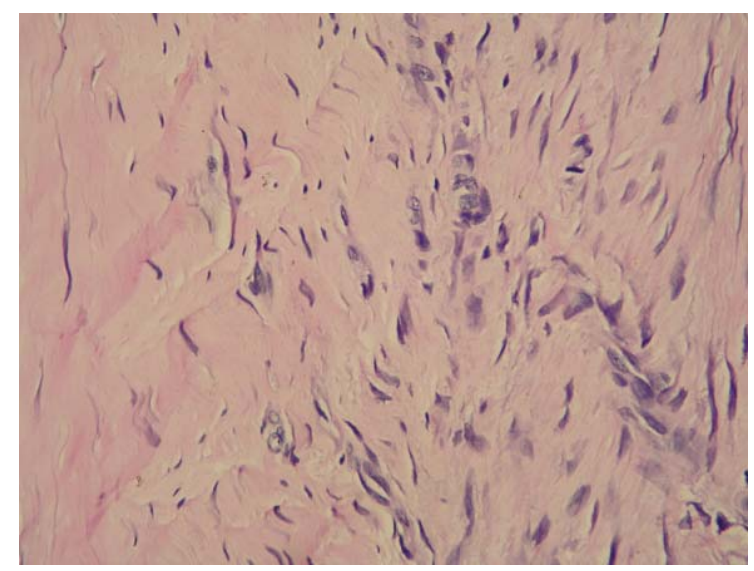

Figure 3. Pleomorphyc nuclei and collagen production in the stroma of the tumor. H\&E staining, orig. magn. $10 \times 40$

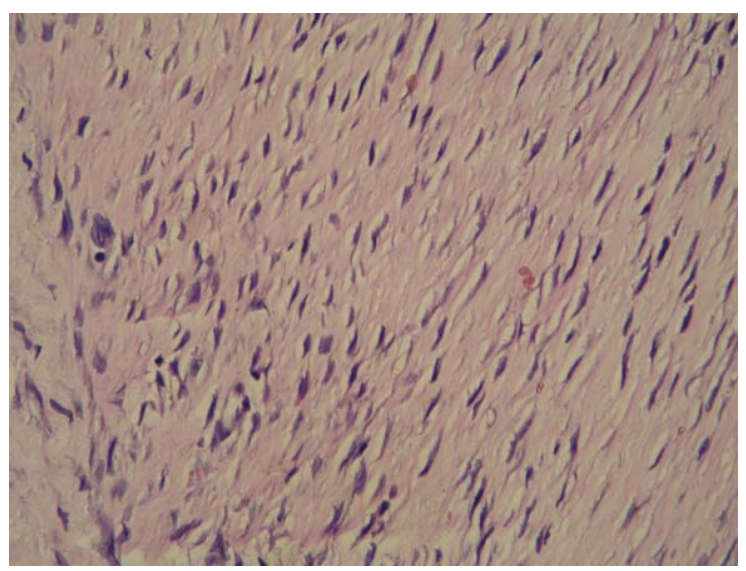

Figure 4. Hypercellular zone at the periphery of the tumour. H\&E staining, orig. magn. 10x40

\section{Discussion}

Fewer than 100 cases have been reported, of which $60 \%$ have been in the metaphysis of the long bones of the appendicular skeleton[7].

$\mathrm{AF}$ is characterized by low mitotic activity and a strong infiltrative growth pattern along tissue planes, with an ability to invade adjacent tissues[1].

The tumours tend to infiltrate adjacent muscle bundles, frequently entrapping them and causing their degeneration. Myofibroblasts are the cells considered to be responsible for the development of desmoid tumours[8].

Musculoaponeurotic fibromatosis is histologically identical to the desmoid tumour.

Though deep or aggressive, fibromatosis is not considered a malignancy. It does not metastasize but multicentric lesions do occur $-[1$, $4,5,9-13]$.

\section{Reference}

1. Jo VY, Fletcher CDM. WHO classification of soft tissue tumours: an update based on the 2013 (4th) edition. Pathology (Phila.). 2014;46(2):95104.

2. Muellar J. Uberden feineren Bau der Krankin often Geschwukste. Berlin: Brecht; 1836.p. 107-13.

3. Macfarlance J. Clinical reports of the surgical practice of the Glasgow Royal Infirmary. Glasgow: Robertson; 1832.p. 63.

4. Li M, Cordon-Cardo C, Gerald WL, Rosai J. Desmoid fibromatosis is a clonal process. Hum Pathol. 1996;27(9):939-43.

5. Sakorafas GH, Nissotakis C, Peros G. Abdominal desmoid tumors. Surg Oncol. 2007;16(2):131-42.

6. Taylor LJ. Musculoaponeurotic fibromatosis. A report of 28 cases and review of the literature. Clin Orthop Relat Res. 1987;224:294-302.

7. de Bree E, Keus R, Melissas J, Tsiftsis D, van Coevorden F. Desmoid tumors: need for an individualized approach. Expert Rev Anticancer Ther. 2009;9(4):525-35

8. Carothers AM, Rizvi H, Hasson RM, Heit YI, Davids JS, Bertagnolli MM, et al. Mesenchymal stromal cell mutations and wound healing contribute to the etiology of desmoid tumors. Cancer Res. 2012;72(1):346-55.

9. Kanyamuhunga A, McCall N, Tuyisenge L, Mumena C, Stefan DC. Aggressive desmoid fibromatosis: First case in a Rwandan child. South Afr J Child Health. 2013;7(3):117-8.

10. Chew NS, Vanhoenacker FM. Aggressive fibromatosis: is PET-CT useful in lesion characterization. JBR-BTR. 2013;96(5):301-3.

11. Kasper B, Strobel P, Hohenberger P. Desmoid Tumors: Clinical Features and Treatment Options for Advanced Disease. Oncologist. 2011; 16(5):682-93.

12. Raju KP, JagadeesanMK, Gururajarao M, Gangadhara DS. Extra-abdominal desmoid tumor of foot: A case report. The Foot and Ankle Online Journal 2014;7(2):1.

13. Ivanov GP, Atanasov DT, Anavi BL. Aggressive juvenile mandibular fibromatosis. Folia Med (Plovdiv). 2013;55(3-4):90-3. 